\title{
SNP marker development in Pinus sylvestris L. in stress-responsive genes characterized from Pinus cembra L. transcriptomes
}

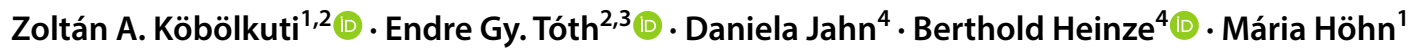

Received: 12 August 2019 / Accepted: 14 May 2020 / Published online: 19 May 2020

(c) The Author(s) 2020

\begin{abstract}
Massively parallel sequencing of cDNA is an efficient route for generating sequence collections that represent expressed genes under different environmental control. The analysis of their sequence helps in developing molecular markers, such as SNPs, which represent a useful tool in detecting adaptive signals in populations. In this study novel PCR markers, based on stress responsive genes, were designed from the transcriptome of the haploxylon Swiss stone pine (Pinus cembra L.) and tested for SNPs in the diploxylon Scots pine (Pinus sylvestris L.). 84 primers were tested on P. sylvestris DNA samples originating from three different types of habitat. After sequencing and BLAST search of the amplified products, parts of 19 different candidate genes were analysed by considering the polymorphic sites, insertions/deletions as well as synonymous and non-synonymous SNPs. In a total of 3735 sites no indels, eight synonymous and 11 non-synonymous SNPs were found. By providing de novo molecular markers developed in P. cembra and tested for transferability in Scots pine, our results give support for the use of de novo markers targeting conserved regions across different pines. The SNPs detected may have important applications in further studies of adaptive genetic variation, providing tools to study relevant genes important in the long-term adaptation of pine species.
\end{abstract}

Keywords Pinus cembra · Pinus sylvestris · Adaptation · Candidate genes · SNP markers

Electronic supplementary material The online version of this article (https://doi.org/10.1007/s11033-020-05527-y) contains supplementary material, which is available to authorized users.

Zoltán A. Köbölkuti

kobolkuti.zoltan@erti.naik.hu;

zoltanattila.kobolkuti@gmail.com

1 Department of Botany, Faculty of Horticulture, Szent István University, Ménesi út 44, Budapest 1118, Hungary

2 Department of Breeding, National Agricultural Research and Innovation Centre, Forest Research Institute, Várkerület 30/A, Sárvár 9600, Hungary

3 Forest Research Institute (IRF), University of Quebec in Abitibi-Témiscamingue UQAT, 445 Boul. de l'Université, Rouyn-Noranda, QC J9X 5E4, Canada

4 Federal Research and Training Centre for Forests, Natural Hazards and Landscape (BFW), Seckendorff-Gudent-Weg 8, 1130 Vienna, Austria

\section{Introduction}

Focus on plant response to environmental stress is becoming increasingly important, as most future climate scenarios suggest climate change that imply an increase in aridity of many areas, causing abiotic stress and seriously threatening natural ecosystems [1]. Aridity influences drought-response trait differentiation and genetic divergence among populations. In these conditions, taking into consideration the genetic information related to adaptation is fundamental in developing conservation strategies [2].

An important way to obtain genetic information is by genome sequencing. Nevertheless, in case of conifers, due to the large genomes, only a few species have been sequenced, so far. This is an obstacle to the genetic evaluation of a large number of species. As an alternative, massively parallel transcriptome sequencing is an efficient route to develop genetic markers [3], which can be used in genetic analysis of different conifer species [4].

Haploxylon Swiss stone pine (Pinus cembra L.), a soft pine, which has one fibrovascular bundle and diploxylon Scots pine (Pinus sylvestris L.), a hard pine, which has two, 
both belonging to subgenus Pinus but belonging to different subsections namely Strobus and Pinus and the latter tending to have harder timber and a larger amount of resin, have formerly been described as highly sensitive to climatic changes $[5,6]$. Scots pine, as a widely tolerant pioneer species, surviving close to the permafrost during the Pleistocene has adapted to different climates, being able to colonize even recently man-disturbed areas [7]. Evaluating genetic variation of the species with focus on the stress-adaptive genes by appropriate genetic tools could provide useful information for the conservation of native remnant populations as a biodiversity resource for the future.

The study was performed to describe (1) homologues in the $P$. cembra transcriptome to formerly annotated stress responsive genes, (2) test on the applicability of primers designed on these gene fragments in P. sylvestris, and (3) genotype by PCR and Sanger sequencing Scots pine samples of different habitat types by revealing possible nucleotide variation at these loci.

\section{Material and methods}

\section{Plant material}

Pinus cembra samples were from a previous comparative study (European larch and Swiss stone pine) (Jahn and Heinze, unpublished), for which material was sampled from six sites along the Austrian Alps (Table S1), 15 individuals from each population. From every tree $2-5$ two-year-old brachyblasts with healthy needles and female cones were collected at four collection stages (June, July, August and September 2015), and male flowers at two stages, in June and July. For RNA extraction, tissues were stored in liquid nitrogen. Among all samples, RNA seq was initially performed on two cones in different developmental stages (collected in June and Sept respectively) and a needle, all three samples originating from one tree in Obergurgel $\left({ }^{\circ} \mathrm{N} 46.86\right.$; ${ }^{\circ} \mathrm{E} 11.01$ ) This transcriptome (as described below) was used in the present study. The primers designed on de novo identified gene sequences were tested in the laboratory on $84 P$. cembra DNA samples, selected at random, all from the collected needles from the six sites previously mentioned. Scots pine samples (10-20 2-year-old brachyblasts with needles/ one individual/population) originated from the Carpathian area, from three natural populations formerly included in a microsatellite study [8] (for details see Table S2), originating from three different types of habitat: wet mountain raised bog from the Eastern Carpathians [Mohos (RO)]; dry rocky outcrop of the lower Tatra region [Kvacany (SK)] and beech-pine mixed forest from the prealpine region of Western Hungary [Csörötnek (HU)], DNA being extracted from one sample/population/habitat type.

\section{De novo identified gene sequences in $P$. cembra}

The most important steps of primer design, testing, sequencing, and analyses of the amplified sequences we highlighted in a flowchart (Fig. 1). RNA was extracted from diploid tissues (scales and needles) by using MasterPure Plant RNA purification Kit (Invitrogen, Epicentre, USA), using the manufacturer's protocol. Messenger RNA was isolated with Dynabeads mRNA DIRECT Micro Kit (ThermoFisher Scientific, Carlsbad, CA, USA) using the manufacturer's protocol, followed by an evaluation according to their RIN value (Bioanalyzer, Agilent, Santa Clara, CA, USA).

Extracted mRNA was used to amplify cDNA libraries, according to the Ion Torrent RNA-Seq protocol (ThermoFisher Scientific, Carlsbad, CA, USA). The RNA Seq analysis was done using an Ion Torrent platform. For each sample a single library was prepared using all reagents and protocols for the Ion Torrent ${ }^{\mathrm{TM}}$ Personal Genome Machine ${ }^{\mathrm{TM}}$ (PGM) System. The quality and quantity of each preparation step was evidenced using a Bioanalyzer Instrument and each library was loaded on a 316 Ion Torrent Chip. After sequencing, further analysis was done using the CLC Bio Genomics Workbench. The output of the library prepared from female cone in the early developmental stage (June) contained 2.1 million reads with a mean read length of $88 \mathrm{bp}$ and a total of $194 \mathrm{Mbp}$. The library created from needles gave a total of $257 \mathrm{Mbp}$, a mean read length of $90 \mathrm{bp}$ and a read count of 2.7 million reads, while the library from the more developed female cone (July) resulted in a mean read length of $83 \mathrm{bp}$, a total of $147 \mathrm{Mbp}$ and around 2.1 million reads. At first, raw reads were quality-trimmed using a quality threshold of 20 . Thereafter, from the library of the female early cone, simple contig sequences were created with a minimum aligned read length of $50 \mathrm{bp}$, medium alignment stringency, word size 20 , bubble size 50 . The de novo assembly of this library resulted in 43,000 contigs with a mean length of $1300 \mathrm{bp}$. Those were used as internal reference in RNA-Seq mapping for the other libraries.

In parallel, data mining was performed, from literature where candidate proteins (enzymes and transcription factors) with possible roles in adaptation were selected. Table S3 presents the candidate proteins selected, based on literature, the presumable coding sequences of which were annotated and PCR cloned during the study.

Based on Table S3, a BLAST database was filled with sequences downloaded from NCBI database (https://www. ncbi.nlm.nih.gov, 07.12.2016), using the following criteria: first, conducting a search for EST and protein sequences for these enzymes and transcription factors that were annotated in the Pinus genus. If such could not be found, the search was extended to "Pinaceae", and if unsuccessful, to "land 
Fig. 1 Diagrammatic representation of the most important steps of gene annotation, primer design and PCR test in $P$. cembra, transfer and sequence analyses during marker development in P. sylvestris

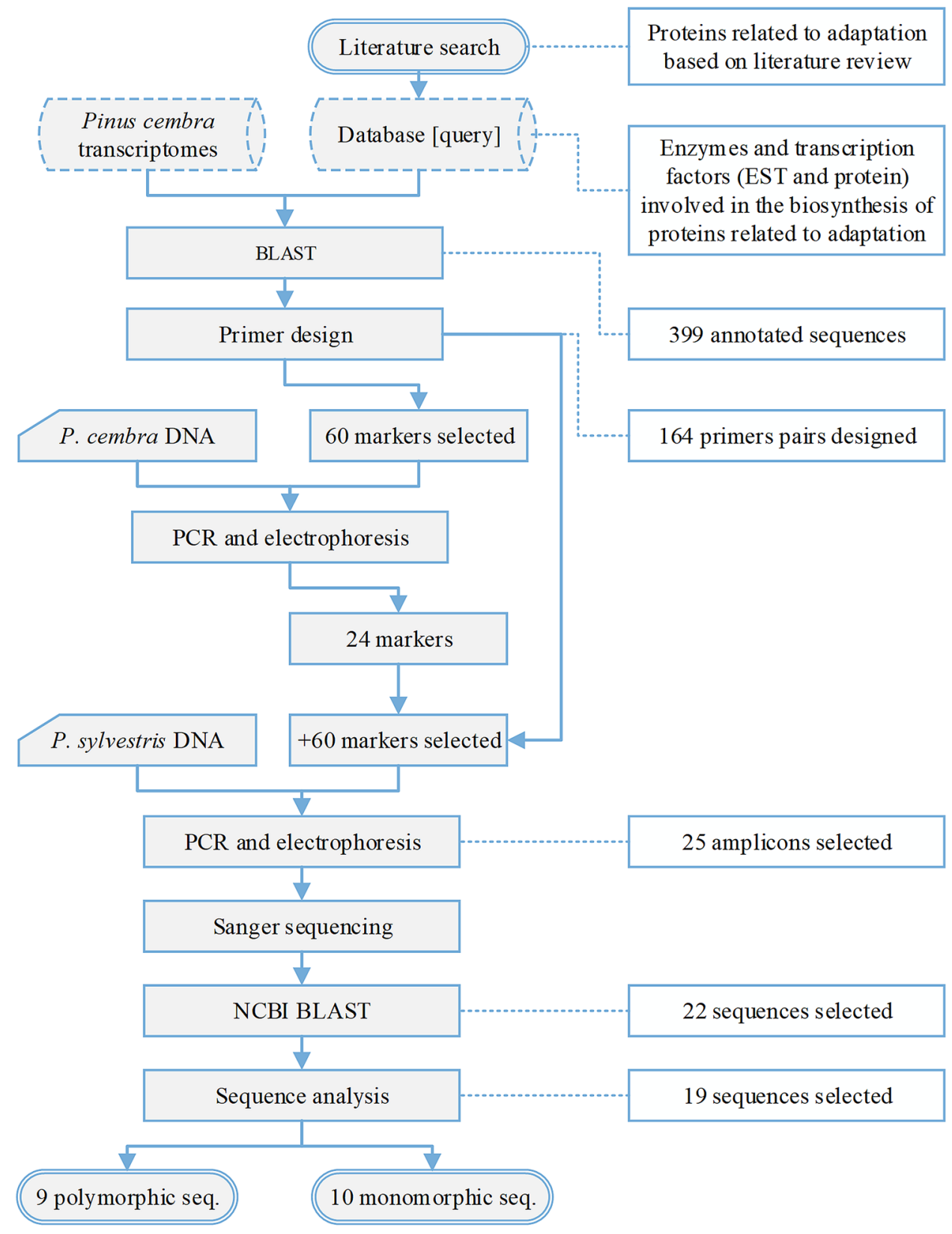

plants". The contents of the BLAST database are presented in Table S4.

De novo assembled contigs were searched using BLASTN and TBLASTN toolkits against the database with CLC Genomic Workbench version 9.0 (QIAGEN Bioinformatics). After the search based on sequence homology, the putative function of the sequences was assigned according to the highest BLAST hits. By this method, from the transcriptome, sequences were selected, annotated and renamed by the coded protein. All sequences were searched again with the BLAST Genome function of the NCBI (https:// blast.ncbi.nlm.nih.gov/Blast.cgi), against the Pinus lambertiana genome (taxid:3343) by BLASTN, optimized for somewhat similar sequences, with algorithm parameters: max target sequences 100, expect threshold 10, word size 11 , max matches in a query range 0 , match/mismatch scores gap costs 2-3 and filtering the low complexity regions and by using a species-specific repeats filter for sugar pine. Pinus lambertiana is the closest sequenced relative of $P$. cembra. This search should indicate any possible introns, as we designed the primers for use with genomic DNA. Primers were designed only on sequences that showed a high degree of similarity and equality in length to translated regions in the $P$. lambertiana genome according to the highest BLAST hits. Following this step, a smaller number of suitable sequences were selected for primer design. The list of the 
designed primer pairs is presented in Table S5. The design of the primers was performed with the primer design toolkit of CLC Genomic Workbench.

To test the amplification, from the newly designed primers, 60 with the most favorable primer selection parameters were selected and tested in the laboratory on 84 randomly selected Swiss stone pine DNA samples (from needles of the six sites previously mentioned in the "Plant material" section), as follows: in a $14 \mu \mathrm{l}$ volume containing $1 \mu \mathrm{l}$ of genomic DNA (about $20 \mathrm{ng}$ ), $0.14 \mu \mathrm{l}$ Polim Phite Taq, GeneAmp PCR buffer II (Applied Biosysytems/Roche, Branchburg, N.J.), $3 \mathrm{mM} \mathrm{MgCl} 2,200 \mu \mathrm{M}$ of dNTPs, $0.2 \mathrm{mM}$ of each primers, by the following PCR (SPECPCR) protocol: denaturation on $94{ }^{\circ} \mathrm{C}$ for $3 \mathrm{~min}(1) ; 60{ }^{\circ} \mathrm{C}$ for $1 \min (2)$, $70{ }^{\circ} \mathrm{C}$ for $1 \mathrm{~min}(3) ; 9 \times$ up to step 3 (4); $94{ }^{\circ} \mathrm{C}$ for $30 \mathrm{~s}(5)$; low stringency annealing $55^{\circ} \mathrm{C}$ for $50 \mathrm{~s}(6) ; 70{ }^{\circ} \mathrm{C}$ for $2 \mathrm{~min}$ (7); 34 cycles up to step 7 (8).

The PCR products were not genotyped by sequencing, being analysed only visually concerning their presence or absence, number and size, by $2 \%$ agarose gel electrophoresis with 3:1 Biogyn Sieve agarose: normal agarose and 1×TAE as electrophoresis buffer.

\section{Test of the designed primers in Scots pine}

DNA extraction from Scots pine samples was done from 20 to $25 \mathrm{mg}$ of plant material of 2-year old needles by using DNeasy Plant Mini Kit (QIAGEN, Valencia, CA, USA) according to the manufacturer's protocol. For testing the newly designed primers, a preliminary PCR test was carried out to evaluate their functionality and transferability. From all primers designed, those that previously amplified only one target in P. cembra and another 60 with optimal primer selection parameters (in total 84 primer pairs) were selected to check for the amplification in Scots pine samples. PCR amplification for this purpose was conducted in a $15 \mu \mathrm{l}$ volume containing $1 \mu \mathrm{l}$ of genomic DNA (about $20 \mathrm{ng}$ ), $0.14 \mu \mathrm{l}$ Polim Phite Taq (1 unit) and, GeneAmp PCR buffer II (Applied Biosysytems/Roche, Branchburg, N.J.), $3 \mathrm{mM} \mathrm{MgCl} 2,200 \mu \mathrm{M}$ of dNTPs, $0.2 \mathrm{mM}$ of each primer, following the PCR protocol: denaturation (1) at $94{ }^{\circ} \mathrm{C}$ for $3 \mathrm{~min}$; followed by (2) $94{ }^{\circ} \mathrm{C}$ for $30 \mathrm{~s}$; annealing (3) at $55^{\circ} \mathrm{C}$ for $0.45 \mathrm{~s}$ and extension (4) at $72{ }^{\circ} \mathrm{C}$ for $1.20 \mathrm{~min}$, (5) $72{ }^{\circ} \mathrm{C}$ for $10 \mathrm{~min}$; the first four cycles being repeated 34 times. The PCR products were analysed by $1 \%$ agarose gel electrophoresis with $1 \times \mathrm{TAE}$ as electrophoresis buffer. Further, only the products that appeared as a single band similar to the expected size (in $P$. cembra) were used to genotype by Sanger sequencing samples with different origin.

For sequencing, the purification of the products after PCR was done by hydrolyzing the excess primers and dephosphorylated unincorporated dNTPs, in one step, with CleanSweep PCR purification reagent (ThermoFisher Scientific,
Carlsbad, CA, USA), according to the manufacturer's protocol. The products resulting from the CleanSweep process were then sequenced using the forward primers, in one direction, at Biomi Ltd., Hungary and at the Institute of Genetics, Biological Research Centre of the Hungarian Academy of Sciences, Szeged, Hungary.

Editing, visualization and alignment of the amplified sequences was accomplished with BioEdit Sequence Alignment Editor version 7.0.9.0 [9] software. Following sequence alignment, genotyping and final validation of all potential SNPs was made visually on chromatograms with use of CodonCode Aligner 8.0.2 (CodonCode Corporation). Number of polymorphic sites, number of insertions/ deletions and the character of SNPs (synonymous or nonsynonymous) was calculated using DNA Sequence Polymorphism v6.10.01 (DNASP) software [10] and validated after a visual check in CodonCode Aligner 8.0.2. Number of synonymous and non-synonymous sites were computed as Nei and Gojobori [11], excluding all cases that go through stop codons. Since our sampling design (one diploid sample/ population) did not make possible the calculation of excess heterozygosity, and our marker development started from P. cembra transcriptomes as well, the paralogous state of our sequences was tested by homology-based search with the list of single- and multi-copy contigs of Rellstab et al. [12], available at Dyriad digital repository: https://doi. org/10.5061/dryad.4bb5849 and the P. sylvestris genome draft [13] with Galaxy (https://usegalaxy.org/) platform's NCBI BLAST + blastn tool (expectation value cutoff 0.001; filtering criteria: the same alignment length as the length of the query) and by transposon search with LTR_FINDER (min LTR length 25, max LTR length 3500, output score threshold 6.0, predicting PBS by using Arabidopsis thaliana L. tRNA database) [14].

\section{Results and discussion}

\section{Screening the $P$. cembra transcriptome data for sequence homologs and testing the markers on $P$. cembra DNA}

Contigs assembled from the three $P$. cembra transcriptomes were screened using BLASTN and TBLASTN toolkits against the BLAST database. From all, 399 sequences were selected, annotated and renamed by the coded protein. The tested 60 primers successfully amplified 42 sequences on 84 different $P$. cembra DNA samples, and 24 PCR products appeared as a single band in the electrophoresis gel (for visual example of three particular amplicons, see Fig S1). Further investigation of these fragments could be a part of a different study concentrating on P. cembra, as we found 
out that they are not present in Rellstab et al.'s lists [12], as detailed below.

\section{Testing the amplified sequences in P. sylvestris}

From all primers producing one band amplicons in Swiss stone pine, complemented as described in the "Material and methods" section and tested in P. sylvestris DNA samples, 53 provided PCR products. 25 PCR products out of these appeared as a single band in the gel (Fig S2). Our study is a PCR primer transferability-SNP discovery test and according to literature, SNP markers from transcriptome-derived sequences already have been developed and utilized in diverse conifer species $[4,15,16]$.

After sequencing, good quality sequences were obtained in case of 22 sequences. A number of three sequences (NAD + 4472, F'3H2550, CHS7594) showed no clear chromatograms, being excluded from further analyses, and CHS381 and MADS1818 showed clear sequencing chromatograms only for PCR products of two samples each. After the homology-based search by BLASTN and BLASTX against the NCBI Genomic Reference Sequences and the NCBI Non-redundant protein seq Database, respectively, in case of three (APX8272, APX637, SOD4683) no significant similarity was found. Accordingly, these sequences were also excluded from further analyses. Table S6 summarizes the results of the BLASTN search against the NCBI Genomic Reference Sequences.

Our results suggest that such primers could work across a wider range of Pinus species. 25 out of 84 primers amplified genomic regions of the expected size, presumably for the reason that these sequences are in interspecies conserved regions of the genus. These type of tests in conifers were reported in several studies like in Hansen et al. [17], Kormutak [18], Chen et al. [19], Liewlaksaneeyanawin et al. [20], Lesser et al. [21] or in Sakaguchi et al. [22].

Our rate of success of transferability was $29.76 \%$, which is lower than that of EST-SSR markers from Pinus taeda to Pinus elliottii var. elliottii (Engelm.) and Pinus caribaea var. hondurensis (Sénécl.) (58\%) [23]. However, taking into consideration that $P$. cembra and $P$. sylvestris are more distant species compared to $P$. elliottii var. elliottii and $P$. caribaea var. hondurensis, this result could be expected.

The results of the sequence analysis of 19 sequences with evaluable chromatograms (in total 4037 sites) using ClustalW and DNA Sequence Polymorphism v6.10.01 are summarized in Table 1. In total, 21 SNPs were found, of which seven alone in Myb 4095. In enzyme coding genes, the number of SNPs was 14 and ranged from one to five. In a second phase of the analysis, the number of indels and the character of SNPs (synonymous or non-synonymous) was determined, the results being summed in Table 1.
Table 1 Outputs of the sequence analysis in P. sylvestris samples with DnaSP v.5.0 effectuated on 55 PCR products amplified by 19 primers

\begin{tabular}{lclll}
\hline Abbrev. & Nr. of sites & $\begin{array}{l}\text { Nr. of poly- } \\
\text { morphic } \\
\text { sites }\end{array}$ & $\begin{array}{l}\text { No. of } \\
\text { non-syn- } \\
\text { onymous } \\
\text { SNPs }\end{array}$ & $\begin{array}{l}\text { No. of } \\
\text { synonymous } \\
\text { SNPs }\end{array}$ \\
\hline Myb 4633 & 297 & 0 & 0 & 0 \\
Myb 4095 & 300 & 7 & 6 & 1 \\
WRKY9928 & 176 & 0 & 0 & 0 \\
WRKY20368 & 207 & 0 & 0 & 0 \\
WRKY4214 & 183 & 0 & 0 & 0 \\
WRKY1289 & 321 & 0 & 0 & 0 \\
MADS15369 & 166 & 1 & 1 & 0 \\
MADS1818* & 300 & 0 & 0 & 0 \\
MADS2038 & 144 & 0 & 0 & 0 \\
MADS12384 & 188 & 1 & 1 & 0 \\
PAL1134 & 177 & 3 & 1 & 2 \\
CHS381* & 301 & 2 & 2 & 0 \\
CHS4014 & 178 & 0 & 0 & 0 \\
F3H350 & 202 & 1 & 0 & 1 \\
IGSTP657 & 263 & 5 & 3 & 2 \\
diTPS11241 & 133 & 0 & 0 & 0 \\
diTPS5871 & 147 & 0 & 0 & 0 \\
APX802 & 277 & 3 & 2 & 1 \\
SOD3685 & 77 & 2 & 1 & 1 \\
Total & 4037 & 21 & 11 & 8 \\
\hline
\end{tabular}

Genes for which only two sequences were analysed are marked with *

Out of 19 sequences, no indels were found. One or two synonymous single nucleotide polymorphisms were found in case of six sequences. Non-synonymous single base mutations ranged from one to six, in case of eight of the analysed sequences. Despite the conserved nature of the primer binding sites, SNP polymorphisms do not seem to be conserved between the samples from the different Scots pine habitat types. This can be tested in a follow-up study.

As a step prior to investigating the polymorphism detected in sequences it is necessary to consider that genes could be pseudogenes, which might lead to incorrect results and false interpretations. Formed by chromosomal duplications or transpositions, these are important features of multi-gene families of large eukaryotic genomes, especially of conifers [24]. By testing the paralogous state of our sequences by homology-based search with the list of single- and multi-copy contigs of Rellstab et al. [11], interestingly, none of our 19 queries were found either among all their released sequences. In our consideration, this fact may be caused by the different sampling design. Two of the specific tissue types (early and mid-stage developing cones) that were used in our study were probably not 
present among the tissue types of Rellstab et al. [12], who used "male and female cones of an adult tree" without specifying their developmental stages in the description. Furthermore, authors used many more tissues and their tree at the Institute location (around Zürich, Switzerland) at unusually low elevation for this species compared to the Obergurgl site at around $2000 \mathrm{~m}$ above sea level in our study may result in different gene expression patterns.

We continued our search by BLASTN against the assembled $P$. sylvestris genome. All queries could be aligned to the draft genome, one candidate (CHS4014) being present at the same alignment length, in two different contigs. Last, we searched our sequences looking for transposable elements using LTR_FINDER. Since no transposons were found and our BLAST results indicated sequence specificity, with only one exception (CHS4014), we conclude that the majority of our designed markers are not pseudogenes.

As variation in relevant genes is essential for the longterm adaptation process, in our consideration, several markers developed here should give interesting new insight in terms of polymorphism. Those that proved to be polymorphic in $P$. sylvestris samples from different habitats, could be used in prospective studies, with large number of individuals, to reveale adaptive divergence between distinct habitat types.

Acknowledgements Open access funding provided by National Agricultural Research and Innovation Centre (NAIK). The authors are grateful to Renate Slunsky and Aleksandra Hilarowicz for the assistance they offered during the laboratory work, and to Wilfried Nebenführ during sampling for P. cembra. The transferability test was supported by National Research, Development and Innovation Office, Hungary by a grant of the Hungarian Scientific Research Fund [OTKA 119208].

Author contributions Zoltán A. Köbölkuti made the database and literature data mining, designed the primers, and made the data analysis. Endre Gy. Tóth has significant contributions to the laboratory work, interpretation of the results and figure editing. Daniela Jahn has major contribution to the preliminary study design, the Swiss stone pine sample collection, material preparation and transcriptome sequencing. Berthold Heinze has significant contributions to the conceptualisation, funding acquisition, institutional support and supervision of the preliminary experiment. Mária Höhn contributed by conceptualization and funding acquisition for the transferability test. The first draft of the manuscript was written by Zoltán A. Köbölkuti and all authors commented on previous versions of the manuscript. All authors read and approved the final manuscript.

\section{Compliance with ethical standards}

Conflict of interest The authors declare that they have no conflict of interest.

Open Access This article is licensed under a Creative Commons Attribution 4.0 International License, which permits use, sharing, adaptation, distribution and reproduction in any medium or format, as long as you give appropriate credit to the original author(s) and the source, provide a link to the Creative Commons licence, and indicate if changes were made. The images or other third party material in this article are included in the article's Creative Commons licence, unless indicated otherwise in a credit line to the material. If material is not included in the article's Creative Commons licence and your intended use is not permitted by statutory regulation or exceeds the permitted use, you will need to obtain permission directly from the copyright holder. To view a copy of this licence, visit http://creativecommons.org/licenses/by/4.0/.

\section{References}

1. Petit JR, Jouzel J, Raynaud D, Barkov NI, Barnola JM, Basile I, Stievenard M (1999) Climate and atmospheric history of the past 420, 000 years from the Vostok ice core, Antartica. Nature 399:429-436. https://doi.org/10.1038/20859

2. Hoban SM, Hauffe HC, Pérez-Espona S, Arntzen JW, Bertorelle G, Bryja J, Frith K, Gaggiotti OE, Galbusera P, Godoy JA, Hoelzel AR, Nichols RA, Primmer CR, Russo IR, Segelbacher G, Siegismund HR, Sihvonen M, Vernesi C, Vilà C, Bruford MW (2013) Bringing genetic diversity to the forefront of conservation policy and management. Conserv Genet Resour 5:593-598. https ://doi.org/10.1007/s12686-013-9859-y

3. Zimin A, Stevens KA, Crepeau MW, Holtz-Morris A, Koriabine M, Marçais G, Puiu D, Roberts M, Wegrzyn JL, De Jong PJ, Neale DB, Salzberg SL, Yorke JA, Langley CH (2014) Sequencing and assembly of the 22-Gb loblolly pine genome. Genetics 196:875-890. https://doi.org/10.1534/genetics.113.159715

4. Parchman TL, Geist KS, Grahnen JA, Benkman CW, Buerkle CA (2010) Transcriptome sequencing in an ecologically important tree species: assembly, annotation, and marker discovery. BMC Genom 11:180. https://doi.org/10.1186/1471-2164-11-180

5. Matías L, Jump S (2012) Interactions between growth, demography and biotic interactions in determining species range limits in a warming world: the case of Pinus sylvestris. For Ecol Manage 282:10-22. https://doi.org/10.1016/j.foreco.2012.06.053

6. Boden S, Pyttel P, Eastaugh CS (2010) Impacts of climate change on the establishment, distribution, growth and mortality of Swiss stone pine (Pinus cembra L.). IForest 3:82

7. Tóth EG, Köbölkuti ZA, Pedryc A, Höhn M (2017) Evolutionary history and phylogeography of Scots pine (Pinus sylvestris L.) in Europe based on molecular markers. J For Res 28:637651. https://doi.org/10.1007/s11676-017-0393-8

8. Tóth EG, Vendramin GG, Bagnoli F, Cseke K, Höhn M (2017) High genetic diversity and distinct origin of recently fragmented Scots pine (Pinus sylvestris L.) populations along the Carpathians and the Pannonian Basin. Tree Genet Genom 13(2):47. https ://doi.org/10.1007/s11295-017-1137-9

9. Hall TA (1999) BioEdit: a user-friendly biological sequence alignment editor and analysis program for Windows 95/98/NT. Nucleic Acids Res 41:95-98

10. Rozas J, Rozas R (1995) DnaSP, DNA sequence polymorphism: an interactive program for estimating population genetics parameters from DNA sequence data. Bioinformatics 11:621625. https://doi.org/10.1093/bioinformatics/11.6.621

11. Nei M, Gojobori T (1986) Simple methods for estimating the numbers of synonymous and nonsynonymous nucleotide substitutions. Mol Biol Evol 3:418-426. https://doi.org/10.1093/ oxfordjournals.molbev.a040410

12. Rellstab C, Dauphin B, Zoller S, Brodbeck S, Gugerli F (2019) Using transcriptome sequencing and pooled exome capture to study local adaptation in the giga-genome of 
Pinus cembra. Mol Ecol Res 19(2):536-551. https://doi. org/10.1111/1755-0998.12986

13. Nystedt B, Street NR, Wetterbom A, Zuccolo A, Lin YC, Scofield DG, Vicedomini R (2013) The Norway spruce genome sequence and conifer genome evolution. Nature 497:579-584

14. Xu Z, Wang H (2007) LTR_FINDER: an efficient tool for the prediction of full-length LTR retrotransposons. Nucleic Acids Res 35:W265-W268. https://doi.org/10.1093/nar/gkm286

15. Pavy N, Gagnon F, Rigault P, Blais S, Deschênes A, Boyle B, Lamothe M (2013) Development of high-density SNP genotyping arrays for white spruce (Picea glauca) and transferability to subtropical and nordic congeners. Mol Ecol 13:324-336

16. Karam MJ, Lefevrè F, Dagher-Kharrat MB, Pinosio S, Vendramin GG (2015) Genomic exploration and molecular marker development in a large and complex conifer genome using RADseq and mRNAseq. Mol Ecol Resour 15:601-612. https:// doi.org/10.1111/1755-0998.12329

17. Hansen OK, Vendramin GG, Sebastiani F, Edwards KJ (2005) Development of microsatellite markers in Abies nordmanniana (Stev.) Spach and cross-species amplification in the Abies genus. Mol Ecol Notes 5:784-787. https://doi.org/10.111 $1 /$ j.1471-8286.2005.01062.x

18. Kormutak A (2004) Crossability relationships between some representatives of the Mediterranean, Northamerican and Asian firs (Abies sp). Veda Publishing House Slovak Academy of Sciences, Bratislava

19. Chen XB, Xie YH, Sun XM (2015) Development and characterization of polymorphic genic-SSR markers in Larix kaempferi.
Molecules 20:6060-6067. https://doi.org/10.3390/molecules2 0046060

20. Liewlaksaneeyanawin C, Ritland C, El-Kassaby Y, Ritland K (2004) Single-copy, species-transferable microsatellite markers developed from loblolly pine ESTs. Theor Appl Genet 109:361369. https://doi.org/10.1007/s00122-004-1635-7

21. Lesser MR, Parchman TL, Buerkle CA (2012) Cross-species transferability of SSR loci developed from transciptome sequencing in lodgepole pine. Mol Ecol Resour 12:448-455. https://doi. org/10.1111/j.1755-0998.2011.03102.x

22. Sakaguchi S, Sugino T, Tsumura Y, Ito M, Crisp MD, Bowman DM, Matsuki Y (2015) High-throughput linkage mapping of Australian white cypress pine (Callitris glaucophylla) and map transferability to related species. Tree Genet Genom 11:121

23. Shepherd M, Cross M, Maguire T, Dieters M, Williams C, Henry R (2002) Transpecific microsatellites for hard pines. Theor Appl Genet 104:819-827. https://doi.org/10.1007/s00122-001-0794-Z

24. Kvarnheden A, Albert VA, Engström P (1998) Molecular evolution of cdc2 pseudogenes in spruce (Picea). Plant Mol Biol 36:767-774. https://doi.org/10.1023/a:1005901413475

Publisher's Note Springer Nature remains neutral with regard to jurisdictional claims in published maps and institutional affiliations. 